\title{
New Spectral Second Kind Chebyshev Wavelets Algorithm for Solving Linear and Nonlinear Second-Order Differential Equations Involving Singular and Bratu Type Equations
}

\author{
W. M. Abd-Elhameed, ${ }^{1,2}$ E. H. Doha, ${ }^{2}$ and Y. H. Youssri ${ }^{2}$ \\ ${ }^{1}$ Department of Mathematics, Faculty of Science, King Abdulaziz University, Jeddah, Saudi Arabia \\ ${ }^{2}$ Department of Mathematics, Faculty of Science, Cairo University, Giza 12613, Egypt \\ Correspondence should be addressed to Y. H. Youssri; youssri@sci.cu.edu.eg
}

Received 27 April 2013; Accepted 31 October 2013

Academic Editor: Turgut Öziş

Copyright (c) 2013 W. M. Abd-Elhameed et al. This is an open access article distributed under the Creative Commons Attribution License, which permits unrestricted use, distribution, and reproduction in any medium, provided the original work is properly cited.

\begin{abstract}
A new spectral algorithm based on shifted second kind Chebyshev wavelets operational matrices of derivatives is introduced and used for solving linear and nonlinear second-order two-point boundary value problems. The main idea for obtaining spectral numerical solutions for these equations is essentially developed by reducing the linear or nonlinear equations with their initial and/or boundary conditions to a system of linear or nonlinear algebraic equations in the unknown expansion coefficients. Convergence analysis and some efficient specific illustrative examples including singular and Bratu type equations are considered to demonstrate the validity and the applicability of the method. Numerical results obtained are compared favorably with the analytical known solutions.
\end{abstract}

\section{Introduction}

Spectral methods are one of the principal methods of discretization for the numerical solution of differential equations. The main advantage of these methods lies in their accuracy for a given number of unknowns (see, e.g., [1$3]$ ). For smooth problems in simple geometries, they offer exponential rates of convergence/spectral accuracy. In contrast, finite difference and finite-element methods yield only algebraic convergence rates. The three most widely used spectral versions are the Galerkin, collocation, and tau methods. Collocation methods have become increasingly popular for solving differential equations, also they are very useful in providing highly accurate solutions to nonlinear differential equations (see, e.g., $[4,5]$ ).

The subject of wavelets has recently drawn a great deal of attention from mathematical scientists in various disciplines. It is creating a common link between mathematicians, physicists, and electrical engineers. Wavelets theory is a relatively new and an emerging area in mathematical research. It has been applied to a wide range of engineering disciplines; particularly, wavelets are very successfully used in signal analysis for wave form representation and segmentations, time frequency analysis, and fast algorithms for easy implementation. Wavelets permit the accurate representation of a variety of functions and operators. Moreover, wavelets establish a connection with fast numerical algorithms (see $[6,7]$ ).

The subject of nonlinear differential equations is a wellestablished part of mathematics, and its systematic development goes back to the early days of the development of calculus. Many recent advances in mathematics, paralleled by a renewed and flourishing interaction between mathematics, sciences, and engineering, have again shown that many phenomena in applied sciences, modelled by differential equations, will yield some mathematical explanation of these phenomena [8-10].

Even-order differential equations have been extensively discussed by a large number of authors due to their great importance in various applications in many fields. For example, in the sequence of papers [1,2,11], the Galerkin method is applied for handling these problems. They constructed suitable basis functions which satisfy the boundary conditions of 
the given differential equation. For this purpose, they used compact combinations of various orthogonal polynomials. The suggested algorithms in these papers are suitable for handling one- and two-dimensional linear high even-order boundary value problems.

In this paper, we aim to give some algorithms for handling both linear and nonlinear second-order boundary value problems based on introducing a new matrix of derivatives, then applying Petrov-Galerkin method on linear equations and collocation method on nonlinear equations. High accurate spectral wavelets solutions are achieved with a small number of retained modes compared with the usual spectral methods, also we can handle singular differential equations with discontinuous variable coefficients.

Among the second-order boundary value problems is the one-dimensional Bratu problem which has a long history. Bratu's own paper appeared in 1914 [12]; generalizations are sometimes called the Liouville-Gelfand or Liouville-GelfandBratu problem in honor of Gel'fand [13] and the nineteenth century work of the great French mathematician Liouville. In recent years, it has been a popular testbed for numerical and perturbation methods [14-16].

Simplification of the solid fuel ignition model in thermal combustion theory yields an elliptic nonlinear partial differential equation, namely, the Bratu problem. Also due to its use in a large variety of applications, many authors have contributed to the study of such problem. Some applications of Bratu problem are the model of thermal reaction process, the Chandrasekhar model of the expansion of the Universe, chemical reaction theory, nanotechnology, and radiative heat transfer (see [17-21]).

The application of Legendre wavelets for solving differential and integral equations is thoroughly considered by many authors (see, [22-26]). Also, Chebyshev wavelets are used for solving some differential, fractional and integral equations (see $[5,27-31])$.

In $[32,33]$, a cosine and sine (CAS) wavelets operational matrix of fractional order integration has been derived and used to solve integrodifferential equations of fractional order.

One approach for solving differential equations is based on converting the differential equations into integral equations through integration, approximating various signals involved in the equation by truncated orthogonal series, and using the operational matrix of integration, to eliminate the integral operations.

Special attentions have been given to applications of block pulse functions [34], Legendre polynomials [35], Chebyshev polynomials [36], Haar wavelets [37], Legendre wavelets [24, 25, 38], and Chebyshev wavelets [27]. Another approach is based on using operational matrix of derivatives in order to reduce the problem into solving a system of algebraic equations (see [23]).

The main aim of this paper is to develop a new spectral algorithm for solving second-order two-point boundary value problems based on shifted second kind Chebyshev wavelets operational matrix of derivatives. The method reduces the differential equation with its initial and/or boundary conditions to a system of algebraic equations in the unknown expansion coefficients. Large systems of algebraic equations may lead to greater computational complexity and large storage requirements. However, the second kind Chebyshev wavelets are structurally sparse; this reduces drastically the computational complexity of solving the resulting algebraic system.

The structure of the paper is as follows. In Section 2, we give some relevant properties of second kind Chebyshev polynomials and their shifted forms. In Section 3, we develop a new shifted second kind Chebyshev wavelets operational matrices of derivatives (SCWOMD); also we ascertain the convergence analysis of the proposed algorithm. As an application of SCWOMD, numerical solutions of second-order linear and nonlinear initial value problems or two-point boundary value problems are implemented and presented in Section 4. In Section 5, some numerical examples are presented to show the efficiency and the applicability of the presented algorithm. Some concluding remarks are given in Section 6.

\section{Some Properties of Second Kind Chebyshev Polynomials and Their Shifted Forms}

In the present section, we discuss some relevant properties of the second kind Chebyshev polynomials and their shifted forms.

2.1. Second Kind Chebyshev Polynomials. It is well known that the second kind Chebyshev polynomials are defined on $[-1,1]$ by

$$
U_{n}(x)=\frac{\sin (n+1) \theta}{\sin \theta}, \quad x=\cos \theta .
$$

These polynomials are orthogonal on $[-1,1]$; that is,

$$
\int_{-1}^{1} \sqrt{1-x^{2}} U_{m}(x) U_{n}(x) d x= \begin{cases}0, & m \neq n, \\ \frac{\pi}{2}, & m=n .\end{cases}
$$

The following properties of second kind Chebyshev polynomials (see, e.g., [39]) are of fundamental importance in the sequel. They are eigenfunctions of the following singular Sturm-Liouville equation:

$$
\left(1-x^{2}\right) D^{2} \phi_{k}(x)-3 x D \phi_{k}(x)+k(k+2) \phi_{k}(x)=0,
$$

where $D \equiv d / d x$ and may be generated by using the recurrence relation

$$
U_{k+1}(x)=2 x U_{k}(x)-U_{k-1}(x), \quad k=1,2,3, \ldots,
$$

starting from $U_{0}(x)=1$ and $U_{1}(x)=2 x$, or from Rodrigues formula

$$
U_{n}(x)=\frac{(-2)^{n}(n+1) !}{(2 n+1) ! \sqrt{1-x^{2}}} D^{n}\left[\left(1-x^{2}\right)^{n+(1 / 2)}\right] .
$$

The following theorem is needed hereafter. 
Theorem 1. The first derivative of second kind Chebyshev polynomials is given by

$$
D U_{n}(x)=2 \sum_{\substack{k=0 \\(k+n) \text { odd }}}^{n-1}(k+1) U_{k}(x) .
$$

For a proof of Theorem 1, see [39].

2.2. Shifted Second Kind Chebyshev Polynomials. The shifted second kind Chebyshev polynomials are defined on $[0,1]$ by $U_{n}^{*}(x)=U_{n}(2 x-1)$. All results of second kind Chebyshev polynomials can be easily transformed to give the corresponding results for their shifted forms. The orthogonality relation with respect to the weight function $\sqrt{x-x^{2}}$ is given by

$$
\int_{0}^{1} \sqrt{x-x^{2}} U_{n}^{*}(x) U_{m}^{*}(x) d x= \begin{cases}0, & m \neq n, \\ \frac{\pi}{8}, & m=n .\end{cases}
$$

The first derivative of $U_{n}^{*}(x)$ is given in the following corollary.

Corollary 2. The first derivative of the shifted second kind Chebyshev polynomial is given by

$$
D U_{n}^{*}(x)=4 \sum_{\substack{k=0 \\(k+n) \text { odd }}}^{n-1}(k+1) U_{k}^{*}(x) .
$$

\section{Shifted Second Kind Chebyshev Operational Matrix of Derivatives}

Wavelets constitute of a family of functions constructed from dilation and translation of single function called the mother wavelet. When the dilation parameter $a$ and the translation parameter $b$ vary continuously, then we have the following family of continuous wavelets:

$$
\psi_{a, b}(t)=|a|^{-1 / 2} \psi\left(\frac{t-b}{a}\right), \quad a, b \in \mathbb{R}, a \neq 0 .
$$

Second kind Chebyshev wavelets $\psi_{n m}(t)=\psi(k, n, m, t)$ have four arguments: $k, n$ can assume any positive integer, $m$ is the order of second kind Chebyshev polynomials, and $t$ is the normalized time. They are defined on the interval $[0,1]$ by

$$
\begin{array}{r}
\psi_{n m}(t)= \begin{cases}\frac{2^{(k+3) / 2}}{\sqrt{\pi}} U_{m}^{*}\left(2^{k} t-n\right), & t \in\left[\frac{n}{2^{k}}, \frac{n+1}{2^{k}}\right], \\
0, & \text { otherwise, }\end{cases} \\
\quad m=0,1, \ldots, M, \quad n=0,1, \ldots, 2^{k}-1 .
\end{array}
$$

3.1. Function Approximation. A function $f(t)$ defined over $[0,1]$ may be expanded in terms of second kind Chebyshev wavelets as

$$
f(t)=\sum_{n=0}^{\infty} \sum_{m=0}^{\infty} c_{n m} \psi_{n m}(t)
$$

where

$$
c_{n m}=\left(f(t), \psi_{n m}(t)\right)_{\omega}=\int_{0}^{1} \omega(t) f(t) \psi_{n m}(t) d t,
$$

and $w(t)=\sqrt{t-t^{2}}$. If the infinite series is truncated, then $f(t)$ can be approximated as

$$
f(t) \simeq \sum_{n=0}^{2^{k}-1} \sum_{m=0}^{M} c_{n m} \psi_{n m}(t)=C^{T} \Psi(t),
$$

where $C$ and $\Psi(t)$ are $2^{k}(M+1) \times 1$ matrices defined by

$$
C=\left[c_{0,0}, c_{0,1}, \ldots, c_{0, M}, \ldots, c_{2^{k}-1, M}, \ldots, c_{2^{k}-1,1}, \ldots, c_{2^{k}-1, M}\right]^{T},
$$

$$
\begin{aligned}
\Psi(t)=[ & \psi_{0,0}, \psi_{0,1}, \ldots, \psi_{0, M}, \ldots, \\
& \left.\psi_{2^{k}-1, M}, \ldots, \psi_{2^{k}-1,1}, \ldots, \psi_{2^{k}-1, M}\right]^{T} .
\end{aligned}
$$

3.2. Convergence Analysis. We state and prove a theorem ascertaining that the second kind Chebyshev wavelet expansion of a function $f(x)$, with bounded second derivative, converges uniformly to $f(x)$.

Theorem 3. A function $f(x) \in L_{\omega}^{2}[0,1]$, with $\left|f^{\prime \prime}(x)\right| \leqslant L$, can be expanded as an infinite sum of Chebyshev wavelets, and the series converges uniformly to $f(x)$. Explicitly, the expansion coefficients in (12) satisfy the following inequality:

$$
\left|c_{n m}\right|<\frac{8 \sqrt{2 \pi} L}{(n+1)^{5 / 2}(m+1)^{2}}, \quad \forall m>1, n \geqslant 0 .
$$

Proof. From (12), it follows that

$$
c_{n m}=\frac{2^{(k+3) / 2}}{\sqrt{\pi}} \int_{n / 2^{k}}^{(n+1) / 2^{k}} f(x) U_{m}^{*}\left(2^{k} x-n\right) \omega\left(2^{k} x-n\right) d x .
$$

If we set $2^{k} x-n=\cos \theta$ in (17), then we get

$$
\begin{aligned}
c_{n m}= & \frac{2^{(-k+3) / 2}}{\sqrt{\pi}} \\
& \times \int_{0}^{\pi} f\left(\frac{\cos \theta+n}{2^{k}}\right) \sin (m+1) \theta \sin \theta d \theta \\
= & \frac{2^{(-k+1) / 2}}{\sqrt{\pi}} \\
& \times \int_{0}^{\pi} f\left(\frac{\cos \theta+n}{2^{k}}\right)[\cos m \theta-\cos (m+2) \theta] d \theta,
\end{aligned}
$$

which gives after integration by parts two times

$$
c_{n m}=\frac{2}{2^{5 k / 2} \sqrt{2 \pi}} \int_{0}^{\pi} f^{\prime \prime}\left(\frac{\cos \theta+n}{2^{k}}\right) \gamma_{m}(\theta) d \theta,
$$


where

$$
\begin{aligned}
\gamma_{m}(\theta)= & \frac{\sin \theta}{m}\left(\frac{\sin (m-1) \theta}{m-1}-\frac{\sin (m+1) \theta}{m+1}\right) \\
& -\frac{\sin \theta}{m+2}\left(\frac{\sin (m+1) \theta}{m+1}-\frac{\sin (m+3) \theta}{m+3}\right) .
\end{aligned}
$$

Therefore, we have

$$
\begin{aligned}
\left|c_{n m}\right| & =\left|\frac{1}{2^{(5 k-1) / 2} \sqrt{\pi}} \int_{0}^{\pi} f^{\prime \prime}\left(\frac{\cos \theta+n}{2^{k}}\right) \gamma_{m}(\theta) d \theta\right| \\
& =\frac{1}{2^{(5 k-1) / 2} \sqrt{\pi}}\left|\int_{0}^{\pi} f^{\prime \prime}\left(\frac{\cos \theta+n}{2^{k}}\right) \gamma_{m}(\theta) d \theta\right| \\
\leqslant & \frac{L}{2^{(5 k-1) / 2} \sqrt{\pi}} \int_{0}^{\pi}\left|\gamma_{m}(\theta)\right| d \theta \\
\leqslant & \frac{L \sqrt{\pi}}{2^{(5 k-1) / 2}} \\
& \times\left[\frac{1}{m}\left(\frac{1}{m-1}+\frac{1}{m+1}\right)\right. \\
& \left.=\frac{1}{2^{(5 k-5) / 2}\left(m^{2}+2 m-3\right)}\left(\frac{1}{m+2}+\frac{1}{m+3}\right)\right] \\
< & \frac{2 L \sqrt{\pi}}{2^{(5 k-5) / 2}(m+1)^{2}} .
\end{aligned}
$$

Since $n \leqslant 2^{k}-1$, we have

$$
\left|c_{n m}\right|<\frac{8 \sqrt{2 \pi} L}{(n+1)^{5 / 2}(m+1)^{2}},
$$

and this completes the proof of Theorem 3.

A shifted second kind Chebyshev wavelets operational matrix of the first derivative is stated and proved in the following theorem.

Theorem 4. Let $\Psi(t)$ be the second kind Chebyshev wavelets vector defined in (15). Then, the first derivative of the vector $\Psi(t)$ can be expressed as

$$
\frac{d \Psi(t)}{d t}=\mathbf{D} \Psi(t)
$$

where $\mathbf{D}$ is $2^{k}(M+1)$ square operational matrix of derivative and is defined by

$$
\mathbf{D}=\left(\begin{array}{cccccc}
F & 0 & . & . & . & 0 \\
0 & F & . & . & . & 0 \\
. & . & . & & . \\
. & . & & . & & . \\
. & . & & . & . \\
0 & 0 & . & . & . & F
\end{array}\right),
$$

in which $F$ is an $(M+1)$ square matrix whose $(r, s)$ th element is defined by

$$
F_{r, s}= \begin{cases}2^{k+2} s, & r \geqslant 2, r>s,(r+s) \text { odd }, \\ 0, & \text { otherwise. }\end{cases}
$$

Proof. If we make use of the shifted second kind Chebyshev polynomials, then the $r$ th element of the vector $\Psi(t)$ defined in (15) can be written in the following form:

$$
\begin{array}{r}
\Psi_{r}(t)=\psi_{n m}(t)=\frac{2^{(k+3) / 2}}{\sqrt{\pi}} U_{m}^{*}\left(2^{k} t-n\right) \chi_{\left[n / 2^{k},(n+1) / 2^{k}\right]}, \\
i=1,2, \ldots, 2^{k}(M+1),
\end{array}
$$

where $r=n(M+1)+(m+1), m=0,1, \ldots, M, n=$ $0,1, \ldots,\left(2^{k}-1\right)$, and

$$
\chi_{\left[n / 2^{k},(n+1) / 2^{k}\right]}= \begin{cases}1, & t \in\left[\frac{n}{2^{k}}, \frac{n+1}{2^{k}}\right], \\ 0, & \text { otherwise. }\end{cases}
$$

If we differentiate (26) with respect to $t$, then we get

$$
\frac{d \Psi_{r}(t)}{d t}=\frac{2^{(2 k+3) / 2}}{\sqrt{\pi}} \frac{d}{d t}\left[U_{m}^{*}\left(2^{k} t-n\right)\right] \chi_{\left[n / 2^{k},(n+1) / 2^{k}\right]} .
$$

It is to be noted here that the R.H.S. of (28) is zero outside the interval $\left[n / 2^{k},(n+1) / 2^{k}\right]$; hence, its second kind Chebyshev wavelets expansion only has those elements in $\Psi(t)$ that are nonzero in the interval $\left[n / 2^{k},(n+1) / 2^{k}\right]$; that is, $\Psi_{i}(t), i=$ $n(M+1)+1, n(M+1)+2, \ldots, n(M+1)+M+1$, and as a consequence, this enables one to expand $\left(d \Psi_{r}(t) / d t\right)$ in terms of shifted second kind Chebyshev wavelets $\Psi_{i}(t), i=n(M+$ $1)+1, n(M+1)+2, \ldots, n(M+1)+M+1$, in the form

$$
\frac{d \Psi_{r}(t)}{d t}=\sum_{i=n(M+1)+1}^{(n+1)(M+1)} a_{i} \Psi_{i}(t),
$$

and accordingly (28) implies that the operational matrix $\mathbf{D}$ is a block matrix defined as in (24). Moreover, we have

$$
\frac{d U_{0}^{*}(t)}{d t}=0
$$

and this implies that

$$
\begin{aligned}
& \frac{d}{d t} \Psi_{r}(t)=0 \\
& \text { for } r=1,(M+1)+1,2(M+1)+1, \ldots, \\
& \qquad\left(2^{k}-1\right)(M+1)+1 .
\end{aligned}
$$

Consequently, the elements of the first row of matrix $F$ defined in (24) are zeros. Now and with the aid of Corollary 2, the first derivative of $\Psi_{r}(t)$ may be expressed in the form

$$
\begin{aligned}
& \frac{d \Psi_{r}(t)}{d t} \\
& =\frac{2^{(2 k+7) / 2}}{\sqrt{\pi}} \sum_{\substack{j=0 \\
(j+m) \text { odd }}}^{m-1}(j+1) U_{j}^{*}\left(2^{k} t-n\right) \chi_{\left[n / 2^{k},(n+1) / 2^{k}\right]} .
\end{aligned}
$$


Expanding this equation in terms of second kind Chebyshev wavelets basis, we get

$$
\frac{d \Psi_{r}(t)}{d t}=2^{k+2} \sum_{\substack{s=0 \\(s+m) \text { odd }}}^{m-1} s \Psi_{n(M+1)+s}(t),
$$

so if $F_{r, s}$ are chosen as

$$
F_{r, s}= \begin{cases}2^{k+2} s, & r=2, \ldots, M+1, s=1, \ldots, r-1, \\ & (r+s) \text { odd }, \\ 0, & \text { otherwise },\end{cases}
$$

then (23) is obtained, and the proof of the theorem is completed.

Corollary 5. The operational matrix for the nth derivative can be obtained from

$$
\frac{d^{n} \Psi(t)}{d t^{n}}=\mathbf{D}^{n} \Psi(t), \quad n=1,2, \ldots
$$

where $\mathbf{D}^{n}$ is the $n$th power of matrix $\mathbf{D}$.

\section{Second-Order Two-Point Boundary Value Problems}

In this section, we are interested in solving linear and nonlinear two-point boundary value problems subject to homogenous or nonhomogenous initial or boundary conditions based on the wavelets operational matrices constructed in Section 3.

4.1. Linear Second-Order Two-Point Boundary Value Problems. Consider the linear second-order differential equation

$$
y^{\prime \prime}(x)+f_{1}(x) y^{\prime}(x)+f_{2}(x) y(x)=g(x), \quad x \in[0,1],
$$

subject to the initial conditions

$$
y(0)=\alpha, \quad y^{\prime}(0)=\beta,
$$

or the boundary conditions

$$
y(0)=\alpha, \quad y(1)=\beta,
$$

or the most general mixed boundary conditions

$$
a_{1} y(0)+a_{2} y^{\prime}(0)=\alpha, \quad b_{1} y(1)+b_{2} y^{\prime}(1)=\beta .
$$

If we approximate $y(x), f_{1}(x), f_{2}(x)$, and $g(x)$ in terms of the second kind Chebyshev wavelets basis, then one can write

$$
\begin{aligned}
& y(x) \simeq \sum_{n=0}^{2^{k}-1} \sum_{m=0}^{M} c_{n m} \psi_{n m}(x)=C^{T} \Psi(x), \\
& f_{1}(x) \simeq \sum_{n=0}^{2^{k}-1} \sum_{m=0}^{M} f_{n m} \psi_{n m}(x)=F_{1}^{T} \Psi(x),
\end{aligned}
$$

$$
\begin{aligned}
& f_{2}(x) \simeq \sum_{n=0}^{2^{k}-1} \sum_{m=0}^{M} \tilde{f}_{n m} \psi_{n m}(x)=F_{2}^{T} \Psi(t), \\
& g(x) \simeq \sum_{n=0}^{2^{k}-1} \sum_{m=0}^{M} g_{n m} \psi_{n m}(x)=G^{T} \Psi(x),
\end{aligned}
$$

where $C^{T}, F_{1}^{T}, F_{2}^{T}$, and $G^{T}$ are defined similarly as in (14). Relations (23) and (35) enable one to approximate $y^{\prime}(x)$ and $y^{\prime \prime}(x)$ as

$$
y^{\prime}(x) \simeq C^{T} \mathrm{D} \Psi(x), \quad y^{\prime \prime}(x) \simeq C^{T} \mathbf{D}^{2} \Psi(x) .
$$

Now, substitution of relations (40) and (41) into (36) enables us to define the residual, $R(x)$, of this equation as

$$
\begin{aligned}
R(x)= & C^{T} \mathbf{D}^{2} \Psi(x)+F_{1}^{T} \Psi(x)(\Psi(x))^{T} \mathbf{D}^{T} C \\
& +F_{2}^{T} \Psi(x)(\Psi(x))^{T} C-G^{T} \Psi(x),
\end{aligned}
$$

and application of the Petrov-Galerkin method (see [40]) yields the following $\left(2^{k}(M+1)-2\right)$ linear equations in the unknown expansion coefficients, $c_{n m}$; namely,

$$
\begin{gathered}
\int_{0}^{1} \sqrt{x-x^{2}} \Psi_{j}(x) R(x) d x=0, \\
\quad j=1,2, \ldots, 2^{k}(M+1)-2 .
\end{gathered}
$$

Moreover, the initial conditions (37), the boundary conditions (38), and the mixed boundary conditions (39) lead, respectively, to the following equations:

$$
\begin{array}{cc}
C^{T} \Psi(0)=\alpha, & C^{T} \mathbf{D} \Psi(0)=\beta, \\
C^{T} \Psi(0)=\alpha, & C^{T} \Psi(1)=\beta, \\
a_{1} C^{T} \Psi(0)+a_{2} C^{T} \mathrm{D} \Psi(0)=\alpha, \\
b_{1} C^{T} \Psi(1)+b_{2} C^{T} \mathrm{D} \Psi(1)=\beta .
\end{array}
$$

Thus, (43) with the two equations of (44) or (45) or (46) generates $2^{k}(M+1)$ a set of linear equations which can be solved for the unknown components of the vector $C$, and hence an approximate spectral wavelets solution to $y(x)$ given in (40) can be obtained.

4.2. Nonlinear Second-Order Two-Point Boundary Value Problems. Consider the nonlinear differential equation

$$
y^{\prime \prime}(x)=F\left(x, y(x), y^{\prime}(x)\right)
$$

subject to the initial conditions

$$
y(0)=\alpha, \quad y^{\prime}(0)=\beta,
$$

or the boundary conditions

$$
y(0)=\alpha, \quad y(1)=\beta,
$$

or the most general mixed boundary conditions

$$
a_{1} y(0)+a_{2} y^{\prime}(0)=\alpha, \quad b_{1} y(1)+b_{2} y^{\prime}(1)=\beta .
$$


If we follow the same procedure of Section 4.1, approximate $y(x)$ as in (40) and make use of (23) and (35), then we obtain

$$
C^{T} \mathbf{D}^{2} \Psi(x)=F\left(x, y(x), C^{T} \mathbf{D} \Psi(x)\right) .
$$

To find an approximate solution to $y(x)$, we compute (51) at the first $2^{k}(M+1)-2$ roots of $U_{2^{k}(M+1)}^{*}(x)$. These equations with (44) or (45) or (46) generate $2^{k}(M+1)$ nonlinear equations in the expansion coefficients, $c_{n m}$, which can be solved with the aid of Newton's iterative method.

\section{Numerical Results and Discussions}

In this section, the presented algorithms in Section 4 are applied to solve linear and nonlinear second-order initial and boundary value problems as well as Bratu's equations. Some examples subject to different initial, boundary, and mixed boundary conditions are considered to illustrate the efficiency and the applicability of the proposed algorithms. All computations are performed by using Mathematica 8 .

Example 1. Consider the following linear second-order boundary value problem:

$$
\begin{array}{r}
y^{\prime \prime}(x)+(1-x) y^{\prime}(x)+2 y(x)=\left(1+2 x-x^{2}\right) \sin x, \\
0<x<1, \quad y(0)=1, \quad y(1)=0,
\end{array}
$$

with the exact solution $y(x)=(1-x) \cos x$.

In Table 1, the maximum absolute error $E$ is listed for various values of $k$ and $M$.

Example 2. Consider the following linear second-order boundary value problem:

$$
y^{\prime \prime}(x)-\sin x y^{\prime}(x)+y(x)=\sin ^{2} x ; \quad 0<x<1,
$$

subject to the mixed boundary conditions

$$
y(0)+y^{\prime}(0)=1, \quad \sin (1) y(1)+\cos (1) y^{\prime}(1)=0,
$$

with the exact solution $y(x)=\cos x$.

In Table 2, the maximum absolute error $E$ is listed for various values of $k$ and $M$.

Example 3. Consider the following nonlinear initial value problem (see [41]):

$$
\begin{gathered}
4 y^{\prime \prime}(x)-2\left(y^{\prime}(x)\right)^{2}+y(x)=0 \\
0<x<1, \quad y(0)=-1, \quad y^{\prime}(0)=0
\end{gathered}
$$

with the exact solution $y(x)=\left(x^{2} / 8\right)-1$.

We solve (55) using the algorithm described in Section 4.2 for the case corresponds to $M=2, k=0$ to obtain an approximate solution of $y(x)$. First, if we make use of (24) and
TABLe 1: Maximum absolute error $E$ for Example 1.

\begin{tabular}{cccccc}
\hline$k$ & $M$ & $E$ & $k$ & $M$ & $E$ \\
\hline \multirow{3}{*}{0} & 3 & $2.28 \cdot 10^{-3}$ & & 2 & $9.23 \cdot 10^{-6}$ \\
& 4 & $6.32 \cdot 10^{-5}$ & 1 & 3 & $8.62 \cdot 10^{-8}$ \\
& 5 & $6.23 \cdot 10^{-6}$ & & 4 & $1.07 \cdot 10^{-9}$ \\
\hline
\end{tabular}

TABle 2: Maximum absolute error $E$ for Example 2.

\begin{tabular}{cccccc}
\hline$k$ & $M$ & $E$ & $k$ & $M$ & $E$ \\
\hline & 3 & $2.52 \cdot 10^{-5}$ & & 2 & $4.90 \cdot 10^{-8}$ \\
0 & 4 & $2.11 \cdot 10^{-6}$ & 1 & 3 & $2.55 \cdot 10^{-9}$ \\
& 5 & $9.01 \cdot 10^{-8}$ & & 4 & $3.50 \cdot 10^{-10}$ \\
\hline
\end{tabular}

(35), then the two operational matrices $\mathbf{D}$ and $\mathbf{D}^{2}$ are given, respectively, by

$$
\mathbf{D}=\left(\begin{array}{ccc}
0 & 0 & 0 \\
4 & 0 & 0 \\
0 & 8 & 0
\end{array}\right), \quad \mathbf{D}^{2}=\left(\begin{array}{ccc}
0 & 0 & 0 \\
0 & 0 & 0 \\
32 & 0 & 0
\end{array}\right)
$$

Moreover, $\Psi(x)$ can be evaluated to give

$$
\Psi(x)=\sqrt{\frac{2}{\pi}}\left(\begin{array}{c}
2 \\
8 x-4 \\
32 x^{2}-32 x+6
\end{array}\right) .
$$

If we set

$$
C=\left(c_{0,0}, c_{0,1}, c_{0,2}\right)^{T}=\sqrt{\frac{\pi}{2}}\left(c_{0}, c_{1}, c_{2}\right)^{T},
$$

then (55) takes the form

$$
4 C^{T} \mathbf{D}^{2} \Psi(x)-2\left(C^{T} \mathbf{D} \Psi(x)\right)^{2}+C^{T} \Psi(x)=0,
$$

which is equivalent to

$$
\begin{aligned}
c_{0} & -2 c_{1}+131 c_{2}-64 c_{1}^{2}+512 c_{1} c_{2}-1024 c_{2}^{2} \\
& +4\left(c_{1}-4 c_{2}-256 c_{1} c_{2}+1024 c_{2}^{2}\right) x \\
& +16\left(c_{2}-256 c_{2}^{2}\right) x^{2}=0 .
\end{aligned}
$$

We only need to satisfy this equation at the first root of $U_{3}^{*}(x)$, that is, at $x_{1}=(2-\sqrt{2}) / 4$, to get

$$
c_{0}-64 c_{1}^{2}+\sqrt{2} c_{1}\left(256 c_{2}-1\right)+\left(129-512 c_{2}\right) c_{2}=0 .
$$

Furthermore, the use of initial conditions in (55) leads to the two equations

$$
\begin{gathered}
2 c_{0}-4 c_{1}+6 c_{2}=-1, \\
c_{1}-4 c_{2}=0 .
\end{gathered}
$$

The solution of the nonlinear system of (61)-(62) gives

$$
c_{0}=\frac{-123}{256}, \quad c_{1}=\frac{1}{64}, \quad c_{2}=\frac{1}{256},
$$


and consequently

$$
\begin{aligned}
y(x) & =\left(\frac{-123}{256}, \frac{1}{64}, \frac{1}{256}\right)\left(\begin{array}{c}
2 \\
8 x-4 \\
32 x^{2}-32 x+6
\end{array}\right) \\
& =\frac{x^{2}}{8}-1,
\end{aligned}
$$

which is the exact solution.

Example 4. Consider the following nonlinear second-order boundary value problem:

$$
\begin{array}{r}
y^{\prime \prime}(x)+\left(y^{\prime}(x)\right)^{2}-y(x)=\sinh ^{2} x, \\
y(0)=1, \quad y(1)=\cosh 1,
\end{array}
$$

with the exact solution $y(x)=\cosh x$.

In Table 3, we list the maximum absolute error $E$, for various values of $k$ and $M$.

Example 5. Consider the following linear singular initial value problem (see [23]):

$$
\begin{array}{r}
y^{\prime \prime}(x)+f(x) y^{\prime}(x)+y(x)=g(x), \\
y(0)=0, \quad y^{\prime}(0)=1,
\end{array}
$$

where

$$
f(x)= \begin{cases}-4 x+\frac{4}{3}, & 0 \leqslant x<\frac{1}{4} \\ x-\frac{1}{3}, & \frac{1}{4} \leqslant x \leqslant 1\end{cases}
$$

and $g(x)$ is chosen such that the exact solution of (66) is $y(x)=e^{x} \sin x /\left(1+x^{2}\right)$. We apply the algorithm described in Section 4.1 with $k=2$. In Table 4 , we list the maximum absolute error for various values of $M$, while in Figure 1, the exact solution is compared with the three numerical solutions corresponding to $k=2, M=2, k=2, M=4, k=2$, and $M=6$ and also in Table 5 we give a comparison between the error obtained in [23] and the present method, $k=2, M=8$.

Example 6. Consider the following Bratu equation (see [1720]):

$$
y^{\prime \prime}(x)+\lambda e^{y(x)}=0, \quad y(0)=y(1)=0,0 \leqslant x \leqslant 1,
$$

with the analytical solution

$$
y(x)=-2 \ln \left[\frac{\cosh ((\theta / 4)(2 x-1))}{\cosh (\theta / 4)}\right]
$$

where $\theta$ is the solution of the nonlinear equation $\theta=$ $\sqrt{2 \lambda} \cosh \theta$. The presented algorithm in Section 4.2 is applied to numerically solve (68), for the three cases corresponding to $\lambda=1,2$ and 3.51 which yield $\theta=1.51716,2.35755$, and 4.66781 , respectively. In Table 6 , the maximum absolute error $E$ is listed for $k=0$ and various values of $M$, while in Table 7 ,

\begin{tabular}{|c|c|c|c|c|c|c|}
\hline$M$ & $\lambda$ & $E$ & $\lambda$ & $E$ & $\lambda$ & $E$ \\
\hline 10 & \multirow{6}{*}{1} & $2.162 \cdot 10^{-7}$ & \multirow{6}{*}{2} & $2.758 \cdot 10^{-5}$ & \multirow{6}{*}{3.51} & $7.658 \cdot 10^{-4}$ \\
\hline 12 & & $4.453 \cdot 10^{-9}$ & & $8.082 \cdot 10^{-6}$ & & $8.665 \cdot 10^{-5}$ \\
\hline 14 & & $8.461 \cdot 10^{-11}$ & & $1.461 \cdot 10^{-9}$ & & $2.384 \cdot 10^{-8}$ \\
\hline 16 & & $1.546 \cdot 10^{-12}$ & & $5.342 \cdot 10^{-10}$ & & $6.587 \cdot 10^{-9}$ \\
\hline 18 & & $2.782 \cdot 10^{-14}$ & & $1.644 \cdot 10^{-11}$ & & $2.387 \cdot 10^{-10}$ \\
\hline 20 & & $7.216 \cdot 10^{-16}$ & & $4.024 \cdot 10^{-12}$ & & $1.058 \cdot 10^{-10}$ \\
\hline
\end{tabular}
we give a comparison between the best errors obtained by
TABle 3: Maximum absolute error $E$ for Example 4.

\begin{tabular}{cccccc}
\hline$k$ & $M$ & $E$ & $k$ & $M$ & $E$ \\
\hline 0 & 3 & $5.69 \cdot 10^{-4}$ & \multirow{2}{*}{1} & 2 & $1.12 \cdot 10^{-6}$ \\
& 4 & $1.35 \cdot 10^{-5}$ & & 3 & $1.97 \cdot 10^{-8}$ \\
\hline
\end{tabular}

TABLE 4: Maximum absolute error $E$ for Example 5.

\begin{tabular}{lccc}
\hline$M$ & $E$ & $M$ & $E$ \\
\hline 2 & $2.98 \cdot 10^{-4}$ & 6 & $1.85 \cdot 10^{-10}$ \\
4 & $1.70 \cdot 10^{-5}$ & 8 & $9.12 \cdot 10^{-11}$ \\
\hline
\end{tabular}

TABLE 5: Comparison between different errors for Example 5.

\begin{tabular}{lcccc}
\hline$x$ & 0.2 & 0.4 & 0.6 & 0.8 \\
\hline $\begin{array}{l}\text { Legendre } \\
\text { Wavelets [23] }\end{array}$ & $4.30 \cdot 10^{-12}$ & $1.24 \cdot 10^{-11}$ & $2.97 \cdot 10^{-12}$ & $4.35 \cdot 10^{-12}$ \\
SCWOMD & $6.47 \cdot 10^{-13}$ & $1.21 \cdot 10^{-12}$ & $1.74 \cdot 10^{-12}$ & $2.32 \cdot 10^{-12}$ \\
\hline
\end{tabular}

TABle 6: Maximum absolute error $E$ for Example 6.

TABLE 7: Comparison between the best errors for Example 6 for $k=$ 0 .

SCWOMD Error in [17] Error in [18] Error in [19] Error in [20] \begin{tabular}{lllll}
\hline $7.22 \cdot 10^{-16}$ & $1.02 \cdot 10^{-6}$ & $8.89 \cdot 10^{-6}$ & $1.35 \cdot 10^{-5}$ & $3.01 \cdot 10^{-3}$
\end{tabular}

our method and some other numerical methods used to solve Example 6. This table shows that our method is more accurate if compared with the methods developed in [1720]. In addition, Figure 2 illustrates a comparison between different solutions obtained by our algorithm in case of $\lambda=1$, $k=0$, and $M=1,2,3$.

Remark 6. It is worth noting here that the obtained numerical results in the previous six solved examples are very accurate, although the number of retained modes in the spectral expansion is very few, and again the numerical results are compared favorably with the known analytical solutions.

\section{Concluding Remarks}

In this paper, an algorithm for obtaining a numerical spectral solution for second-order linear and nonlinear boundary value problems is discussed. The derivation of this algorithm is essentially based on constructing the shifted second kind Chebyshev wavelets operational matrix of differentiation. One of the main advantages of the presented algorithm is its availability for application on both linear and nonlinear second-order boundary value problems including some 


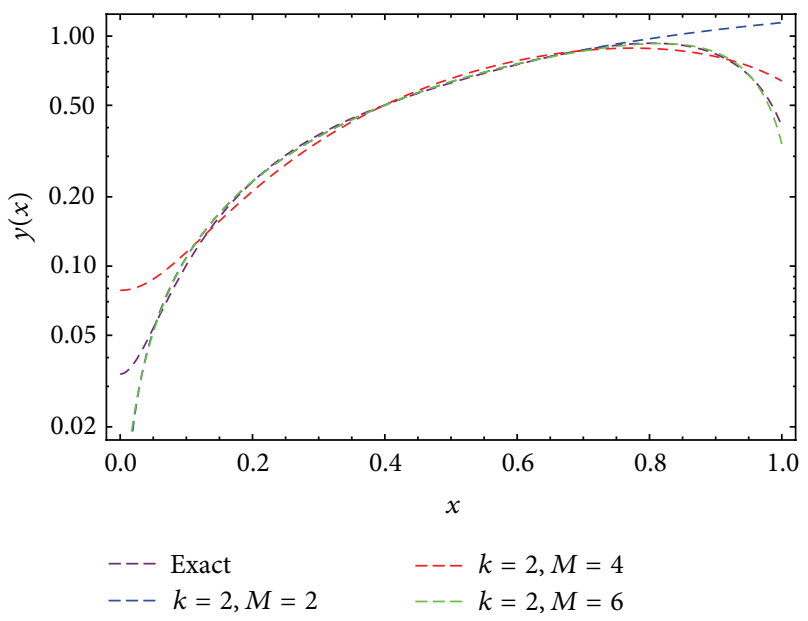

Figure 1: Numerical and exact solutions of Example 5.

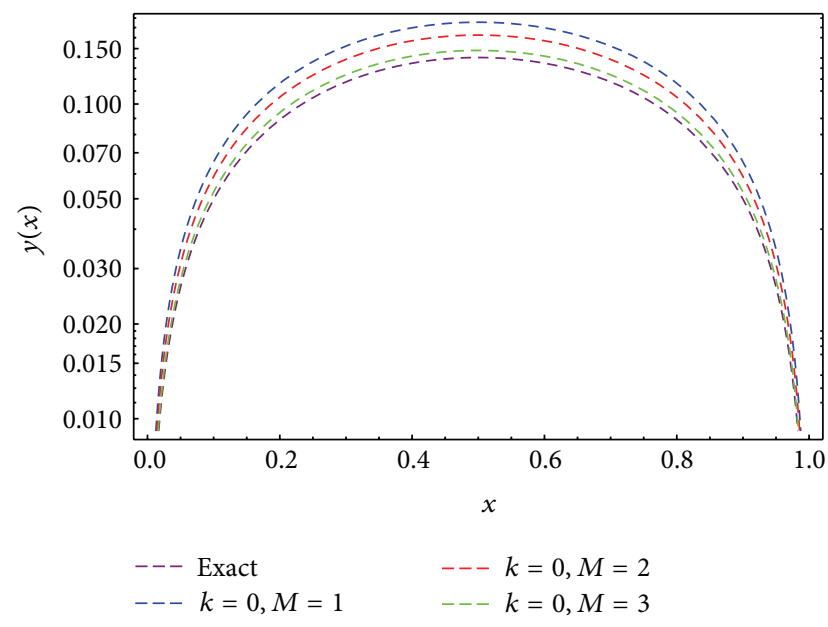

Figure 2: Different solutions of Example 6.

singular equations and also Bratu type equations. Another advantage of the developed algorithm is that high accurate approximate solutions are achieved using a small number of the second kind Chebyshev wavelets. The obtained numerical results are compared favorably with the analytical ones.

\section{Acknowledgments}

This paper was funded by the Deanship of Scientific Research (DSR), King Abdulaziz University, Jeddah. The authors, therefore, acknowledge with thanks DSR technical and financial support. Also, they would like to thank the referee for his valuable comments and suggestions which improved the paper into its present form.

\section{References}

[1] E. H. Doha and W. M. Abd-Elhameed, "Efficient spectral-Galerkin algorithms for direct solution of second-order equations using ultraspherical polynomials," SIAM Journal on Scientific Computing, vol. 24, no. 2, pp. 548-571, 2002.
[2] E. H. Doha, W. M. Abd-Elhameed, and M. A. Bassuony, "New algorithms for solving high even-order differential equations using third and fourth Chebyshev-Galerkin methods," Journal of Computational Physics, vol. 236, pp. 563-579, 2013.

[3] W. M. Abd-Elhameed, E. H. Doha, and Y. H. Youssri, "Efficient spectral-Petrov-Galerkin methods for third- and fifth-order differential equations using general parameters generalized Jacobi polynomials," Quaestiones Mathematicae, vol. 36, no. 1, pp. 15-38, 2013.

[4] E. H. Doha, W. M. Abd-Elhameed, and Y. H. Youssri, "Second kind Chebyshev operational matrix algorithm for solving differential equations of Lane-Emden type," New Astronomy, vol. 23-24, pp. 113-117, 2013.

[5] W. M. Abd-Elhameed, E. H. Doha, and Y. H. Youssri, "New wavelets collocation method for solving second-order multipoint boundary value problems using Chebyshev polynomials of third and fourth kinds," Abstract and Applied Analysis, vol. 2013, Article ID 542839, 9 pages, 2013.

[6] A. Constantmldes, Applied Numerical Methods With Personal Computers, McGraw-Hill, New York, NY, USA, 1987.

[7] D. E. Newland, An Introduction to Random Vibrations, Spectral and Wavelet Analysis, Longman Scientific and Technical, New York, NY, USA, 1993.

[8] M. Pašić, "Fite-Wintner-Leighton-type oscillation criteria for second-order differential equations with nonlinear damping," Abstract and Applied Analysis, vol. 2013, Article ID 852180, 10 pages, 2013.

[9] N. Daoudi-Merzagui, F. Derrab, and A. Boucherif, "Subharmonic solutions of nonautonomous second order differential equations with singular nonlinearities," Abstract and Applied Analysis, vol. 2012, Article ID 903281, 20 pages, 2012.

[10] M. Turkyilmazoglu, "Effective computation of exact and analytic approximate solutions to singular nonlinear equations of Lane-Emden-Fowler type," Applied Mathematical Modelling, vol. 37, pp. 7539-7548, 2013.

[11] E. H. Doha and W. M. Abd-Elhameed, "Efficient solutions of multidimensional sixth-order boundary value problems using symmetric generalized Jacobi-Galerkin method," Abstract and Applied Analysis, vol. 2012, Article ID 749370, 19 pages, 2012.

[12] G. Bratu, "Sur les équations intégrales non linéaires," Bulletin de la Société Mathématique de France, vol. 42, pp. 113-142, 1914.

[13] I. M. Gel'fand, "Some problems in the theory of quasilinear equations," American Mathematical Society Translations, vol. 29, pp. 295-381, 1963.

[14] A. Serghini Mounim and B. M. de Dormale, "From the fitting techniques to accurate schemes for the Liouville-Bratu-Gelfand problem," Numerical Methods for Partial Differential Equations, vol. 22, no. 4, pp. 761-775, 2006.

[15] M. I. Syam and A. Hamdan, "An efficient method for solving Bratu equations," Applied Mathematics and Computation, vol. 176, no. 2, pp. 704-713, 2006.

[16] A.-M. Wazwaz, "Adomian decomposition method for a reliable treatment of the Bratu-type equations," Applied Mathematics and Computation, vol. 166, no. 3, pp. 652-663, 2005.

[17] S. Abbasbandy, M. S. Hashemi, and C.-S. Liu, "The Lie-group shooting method for solving the Bratu equation," Communications in Nonlinear Science and Numerical Simulation, vol. 16, no. 11, pp. 4238-4249, 2011.

[18] H. Caglar, N. Caglar, M. Özer, A. Valarıstos, and A. N. Anagnostopoulos, "B-spline method for solving Bratu's problem," International Journal of Computer Mathematics, vol. 87, no. 8, pp. 1885-1891, 2010. 
[19] S. A. Khuri, "A new approach to Bratu's problem," Applied Mathematics and Computation, vol. 147, no. 1, pp. 131-136, 2004.

[20] E. Deeba, S. A. Khuri, and S. Xie, "An algorithm for solving boundary value problems," Journal of Computational Physics, vol. 159, no. 2, pp. 125-138, 2000.

[21] J. P. Boyd, "One-point pseudospectral collocation for the onedimensional Bratu equation," Applied Mathematics and Computation, vol. 217, no. 12, pp. 5553-5565, 2011.

[22] H. Jafari, S. A. Yousefi, M. A. Firoozjaee, S. Momani, and C. M. Khalique, "Application of Legendre wavelets for solving fractional differential equations," Computers \& Mathematics with Applications, vol. 62, no. 3, pp. 1038-1045, 2011.

[23] F. Mohammadi and M. M. Hosseini, "A new Legendre wavelet operational matrix of derivative and its applications in solving the singular ordinary differential equations," Journal of the Franklin Institute, vol. 348, no. 8, pp. 1787-1796, 2011.

[24] M. Razzaghi and S. Yousefi, "The Legendre wavelets operational matrix of integration," International Journal of Systems Science, vol. 32, no. 4, pp. 495-502, 2001.

[25] M. Razzaghi and S. Yousefi, "Legendre wavelets method for the solution of nonlinear problems in the calculus of variations," Mathematical and Computer Modelling, vol. 34, no. 1-2, pp. 4554, 2001.

[26] M. Razzaghi and S. Yousefi, "Legendre wavelets method for constrained optimal control problems," Mathematical Methods in the Applied Sciences, vol. 25, no. 7, pp. 529-539, 2002.

[27] E. Babolian and F. Fattahzadeh, "Numerical solution of differential equations by using Chebyshev wavelet operational matrix of integration," Applied Mathematics and Computation, vol. 188, no. 1, pp. 417-426, 2007.

[28] M. Dehghan and A. Saadatmandi, "Chebyshev finite difference method for Fredholm integro-differential equation," International Journal of Computer Mathematics, vol. 85, no. 1, pp. 123130, 2008.

[29] A. Saadatmandi and M. Dehghan, "A new operational matrix for solving fractional-order differential equations," Computers \& Mathematics with Applications, vol. 59, no. 3, pp. 1326-1336, 2010.

[30] L. Zhu and Q. Fan, "Solving fractional nonlinear Fredholm integro-differential equations by the second kind Chebyshev wavelet," Communications in Nonlinear Science and Numerical Simulation, vol. 17, no. 6, pp. 2333-2341, 2012.

[31] Y. Li, "Solving a nonlinear fractional differential equation using Chebyshev wavelets," Communications in Nonlinear Science and Numerical Simulation, vol. 15, no. 9, pp. 2284-2292, 2010.

[32] H. Saeedi, M. Mohseni Moghadam, N. Mollahasani, and G. N. Chuev, "A CAS wavelet method for solving nonlinear Fredholm integro-differential equations of fractional order," Communications in Nonlinear Science and Numerical Simulation, vol. 16, no. 3, pp. 1154-1163, 2011.

[33] H. Saeedi and M. M. Moghadam, "Numerical solution of nonlinear Volterra integro-differential equations of arbitrary order by CAS wavelets," Communications in Nonlinear Science and Numerical Simulation, vol. 16, no. 3, pp. 1216-1226, 2011.

[34] C. F. Cheng, Y. T. Tsay, and T. T. Wu, "Walsh operational matrices for fractional calculus and their application to distributed systems," Journal of the Franklin Institute, vol. 303, no. 3, pp. 267284, 1977.

[35] R. Y. Chang and M. L. Wang, "Shifted Legendre direct method for variational problems," Journal of Optimization Theory and Applications, vol. 39, no. 2, pp. 299-307, 1983.
[36] I. R. Horng and J. H. Chou, "Shifted Chebyshev direct method for solving variational problems," International Journal of Systems Science, vol. 16, no. 7, pp. 855-861, 1985.

[37] J. S. Gu and W. S. Jiang, "The Haar wavelets operational matrix of integration," International Journal of Systems Science, vol. 27, no. 7, pp. 623-628, 1996.

[38] F. Khellat and S. A. Yousefi, "The linear Legendre mother wavelets operational matrix of integration and its application," Journal of the Franklin Institute, vol. 343, no. 2, pp. 181-190, 2006.

[39] J. C. Mason and D. C. Handscomb, Chebyshev Polynomials, Chapman \& Hall/CRC, Boca Raton, Fla, USA, 2003.

[40] C. Canuto, M. Y. Hussaini, A. Quarteroni, and T. A. Zang, Spectral Methods in Fluid Dynamics, Springer Series in Computational Physics, Springer, New York, NY, USA, 1988.

[41] M. R. Eslahchi, M. Dehghan, and S. Ahmadi, "The general Jacobi matrix method for solving some nonlinear ordinary differential equations," Applied Mathematical Modelling, vol. 36, no. 8, pp. 3387-3398, 2012. 


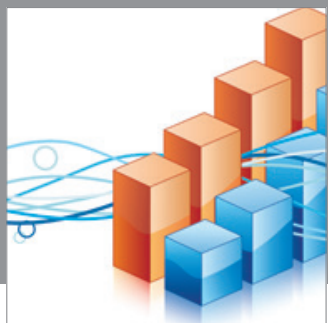

Advances in

Operations Research

mansans

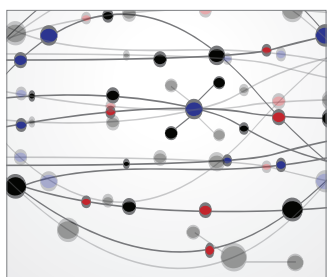

The Scientific World Journal
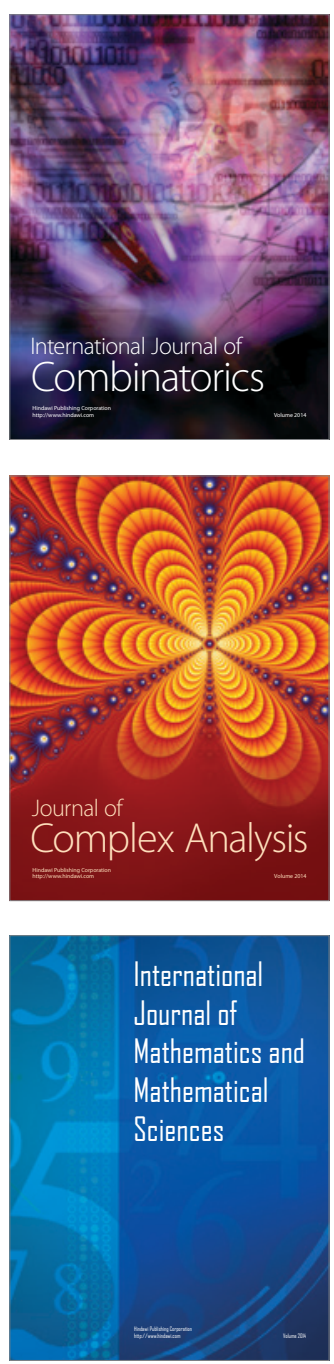
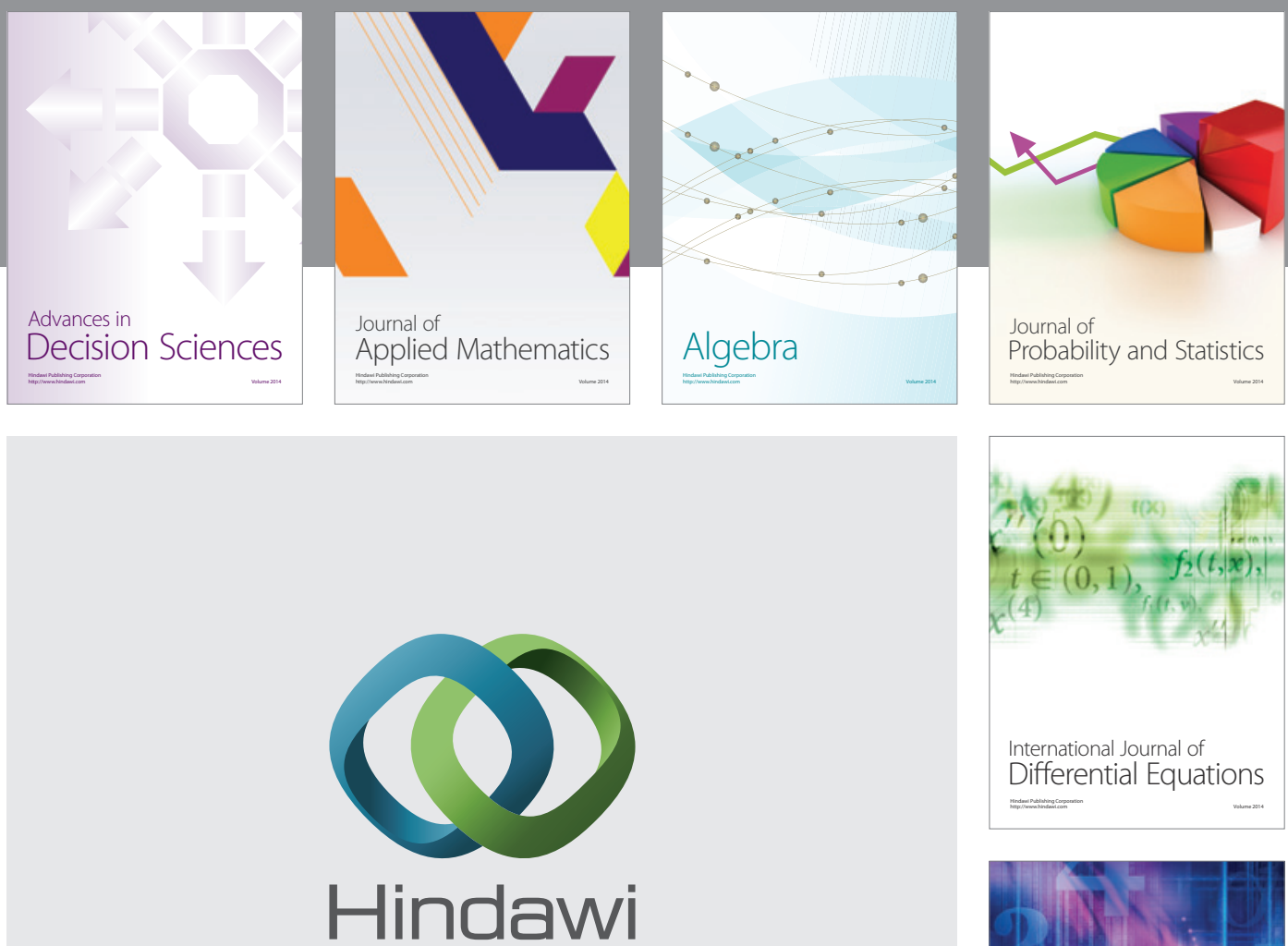

Submit your manuscripts at http://www.hindawi.com
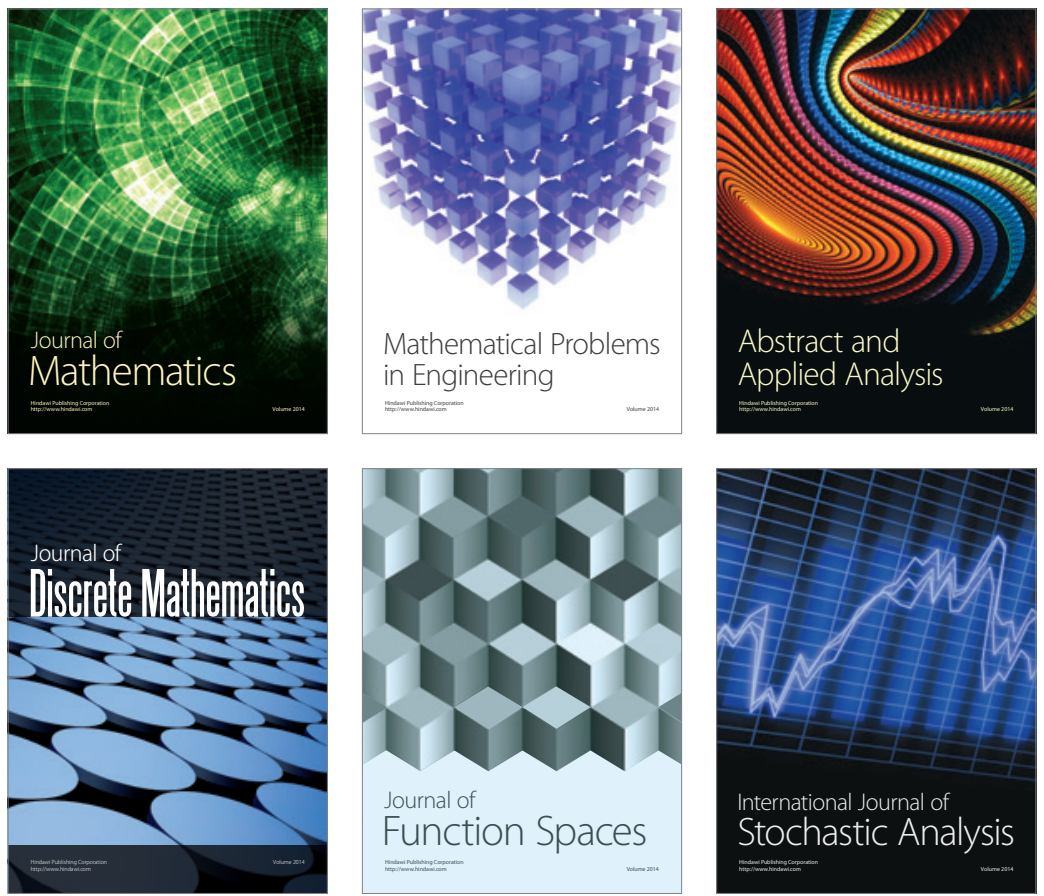

Journal of

Function Spaces

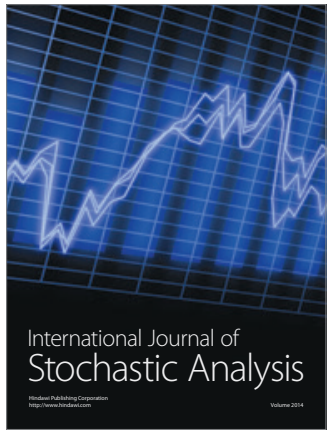

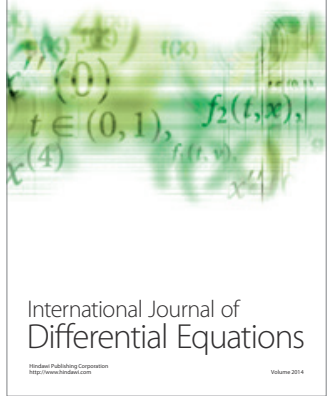
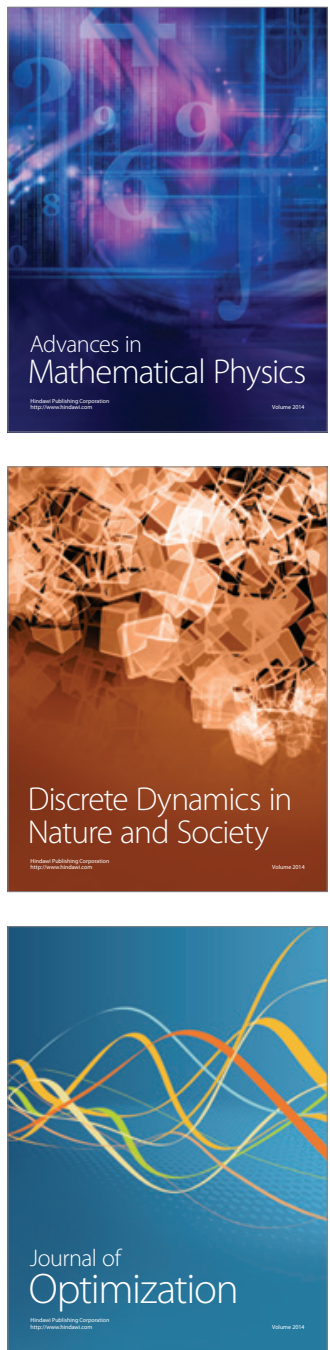